\title{
Intraneural Lipoma with Cartilagenous Metaplasia of Median Nerve
}

\author{
Prashant Kamble, Kalai Vanan, SS Mohanty, Tushar Rathod, Ayushi Sahay \\ Department of Orthopaedics, Seth GS Medical college \& KEM Hospital, Parel, Mumbai; Department of Pathology, Tata Memorial \\ Hospital, Parel, Mumbai, India.
}

\author{
Corresponding Author: \\ Dr. Prashant Kamble \\ Email: dr.prashantkamble@gmail.com \\ This is an Open Access article distributed \\ under the terms of the Creative Commons \\ Attribution License (creativecommons.org/ \\ licenses/by/3.0). \\ Received : April 11, 2017 \\ Accepted : June 19, 2017 \\ Published : September 5, 2017
}

\begin{abstract}
Background: Lipomas are benign tumor, mostly seen in the trunk, nape of the neck, shoulder and upper arm, unusually located in forearm and hands. Lipoma in the forearm may of either in the neural plane or outside the neural plane. Within the neural plane it may be either intra-neural or peri-neural lipoma. Intra-neural lipomas arising from the peripheral nerves are very rare. Case Report: We hereby report intra-neural lipoma with cartilagenous metaplasia of median nerve in distal forearm presenting with carpal tunnel syndrome (CTS) in young female. Conclusion: This case is being reported because of its rare and atypical presentation.
\end{abstract}

Keywords: Carpal Tunnel Syndrome, Hand, Lipoma, Median Nerve, Metaplasia.

\section{Introduction}

Lipomas are benign adipose tissue tumor commonly seen on the trunk and extremities. Lipomas arising from the peripheral nerves are of either intra-neural or peri-neural lipoma. Because of its rare and atypical presentation, we report this case with intra-neural lipoma with cartilaginous metaplasia of median nerve in distal forearm which presented as swelling in distal forearm mimicking carpal tunnel syndrome (CTS) in young female. It started as swelling initially and later presented with symptoms like tingling, numbness on compressing the main nerve trunk.

\section{Case Report}

A 28 year old female presented with insidious onset, gradually progressive swelling in left distal forearm for last one and half year. The patient had tingling and numbness in the median nerve distribution without having weakness in hand. On clinical examination there was a diffuse swelling measuring $5 \times 4 \mathrm{~cm}$ located in the left distal forearm [Fig.1A], minimally tender, firm in consistency. Tinel sign was positive. On investigation, Magnetic
Resonance Imaging (MRI) showed large well defined encapsulated lesion in the inter-muscular and subcutaneous plane with peri-lesional edema without bony invasion compressing and arising from the median nerve [Fig.1B]. Surgical excision of the mass lesion was planned. During intraoperative surgical exploration, there was a well encapsulated yellowish globular mass in the distal forearm arising from median nerve main trunk [Fig.2A]. After meticulous dissection the whole tumor mass [Fig.2B] was removed as en mass. The patient recovered post-operatively, over a period of three weeks, had normal sensation and motor function without any neuro-deficit.

Histopathological and microscopic examination showed yellowish and myxoid mass with scattered lobulated white areas, lesion was composed of benign mature adipocytes, with areas of cartilaginous metaplasia, and interspersed myxoid areas and fibrous bands [Fig.3A,B]. On immunohistochemistry, both the adipocytes and benign cartilage were seen highlighted by S100 protein [Fig.3C,D]. Immunostain for CD34 was negative in the tumor cells. Ki-67 labeling index 
was very low $(<1 \%)$. Histological and immunohistochemical findings are consistent with a lipoma with cartilaginous metaplasia with no malignant changes. On two years of follow up after the surgery, there is no local recurrence of the lesion and with no functional disability in wrist and hand.

\section{Discussion}

Lipomas arising from peripheral nerve are very rare. These are slow growing tumors usually asymptomatic or present as minimal swelling [2]. These are commonly seen in third or fourth decade with female preponderance [2]. In the later stage these tumors cause compression neuropathy. Tumors arising from neural structures includes neurofibroma, schwannomas, and neurotheikioma, or malignant peripheral nerve sheath tumors. The intra-neural tumors originating from neural structures include intra-neural lipoma, fibrofatty tissues, hemangioma and ganglion cysts. According to Terzis JK et al. benign fatty tumors of the peripheral nerve are classified as well-encapsulated intra-neural lipomas, diffusely infiltrating fibrofatty tumors and macrodystrophia lipomatosa [3]. In chondrolipoma (i.e., lipoma with cartilaginous metaplasia) there occurs the presence of mature hyaline cartilaginous cells within the fat tissue. Chondrolipoma are most commonly seen in head and neck region, are treated by surgical excision [4]. Fat cells normally lies between the fascicles of the nerve fibres [5]. However, lipoma arising from this fat cells (intraneural lipoma) are very rare [69]. In 1964, Morely first described intra-neural lipoma [10]. These patients present as mass arising from forearm and sometimes mimicking as carpal tunnel syndrome. Commonest cause of carpal tunnel syndrome is idiopathic. Rarely carpal tunnel syndrome is caused by an space occupying lesion such as ganglion, tendon sheath swelling, perilunate dislocation, distal radius fracture, or lipomas. There is no role of conservative management in symptomatic patients. Complete surgical excision of the tumor mass is warranted. Grossly these tumors show glistening yellowish myxoid areas

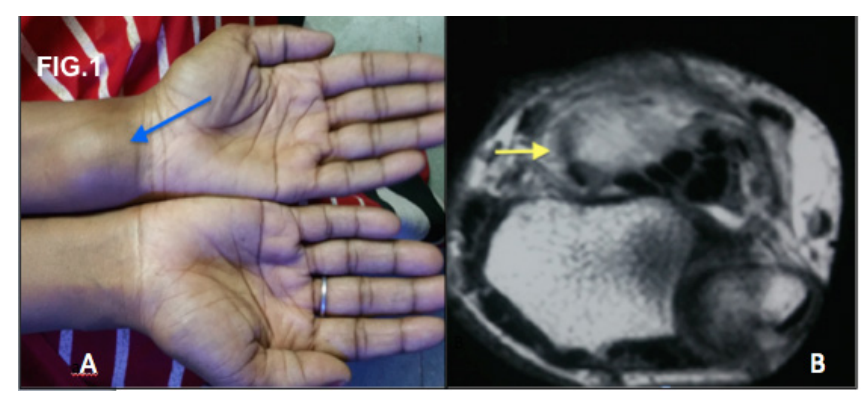

Fig.1(A): Swelling in the left distal one third forearm (blue arrow), (B): MRI showing the mass arising from the median nerve (yellow arrow).
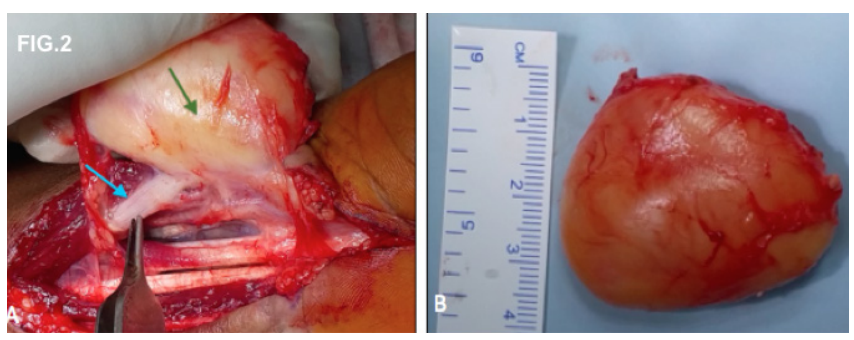

Fig.2(A): Tumor mass (green arrow) attached to the median nerve main trunk (blue arrow), (B): showing whole mass was removed as en mass.

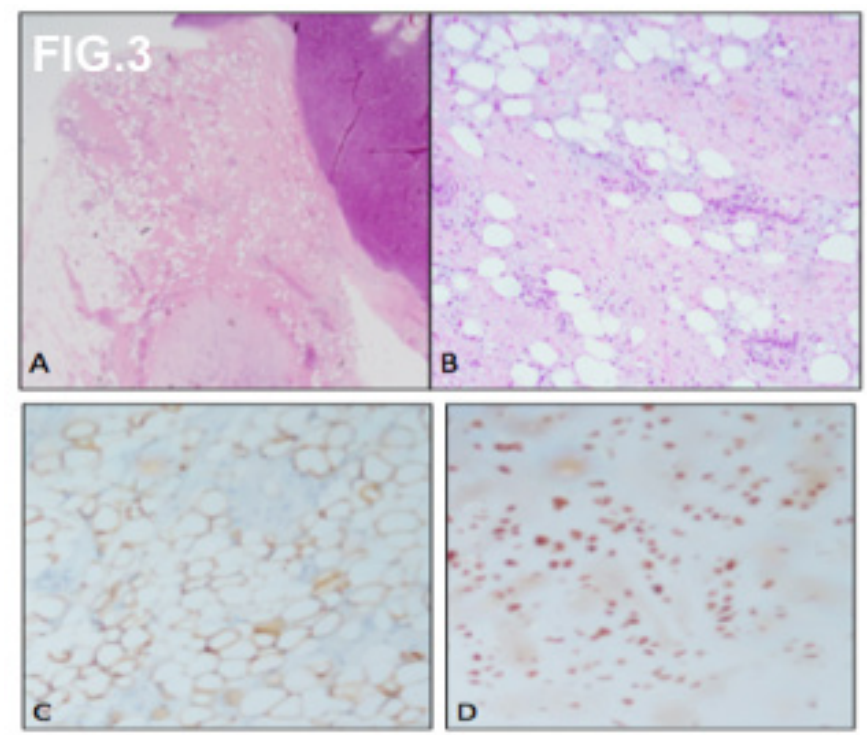

Fig.3(A): Microphotograph showing the encapsulated tumor, composed of mature fat and mature hyaline cartilage (Hematoxylin and eosin, ×40); (B): Microphotograph showing mature adipocytes with interspersed myxoid areas and myxoid change. Scattered fibrous bands are also noted (Hematoxylin and eosin, $\times 40$ ); Immunohistochemical examination showed positivity for $S 100$ in the adipocytes (C): as well and cartilaginous areas (D): $(\times 40)$. 
admixed with white lobules. On microscopic examination these tumors shows mature fat cells. Immunohistochemistry shows S-100 positive for adipocytes. Surgical excision of the whole mass is indicated in symptomatic patients. There is no recurrence of tumor in our case with two years of follow up without any compression neuropathy.

\section{Conclusion}

Considering the limited knowledge due to the paucity of cases reported about the intra-neural lipomas of the peripheral nerves, we report this case because of its rare and atypical presentation.

Contributors: PK: manuscript writing, literature search; KV, SSM: manuscript editing, literature search; TR, AS: Study designing and manuscript editing. PS will act as guarantor. All authors approved the final version of manuscript.

Funding: None; Competing interests: None stated.

\section{References}

1. Flores LP, Carneiro JZ. Peripheral nerve compression secondary to adjacent lipomas. Surg Neurol. 2007;67:258-262.

2. Gennaro S, Merciadri P, Secci F. Intraneural lipoma of the median nerve mimicking carpal tunnel syndrome.
Acta Neurochir (Wien). 2012;154:1299-1301.

3. Terzis JK, Daniel RK, Williams HB, Spencer PS. Benign fatty tumors of the peripheral nerves, Ann Plast Surg, 1978;1:193-216.

4. Shabbir F, Greenwood M. Chondrolipoma presenting as a lump on the lateral tongue. Dent Update. 2011;38:188190.

5. Chatillon $\mathrm{CE}$, Guiot MC, Jacques L. Lipomatous, vascular, and chondromatous benign tumors of the peripheral nerves: representative cases and review of the literature. Neurosurg Focus. 2007;22:E18.

6. Guthikonda M, Rengachary SS, Balko MG, van Loveren H. Lipofibromatous hamartoma of the median nerve: Case report with magnetic resonance imaging correlation, Neurosurgery. 1994;35:127-132.

7. Goldstein LJ, Helfend LK, Kordestani RK. Postoperative edema after vascular access causing nerve compression secondary to the presence of a perineuronal lipoma: Case report. Neurosurgery. 2002;50:412-413.

8. Babins DM, Lubahn JD. Palmar lipomas associated with compression of the median nerve. J Bone Joint Surg Am. 1994;76:1360-1362.

9. Bagatur AE, Yalcinkaya M. Unilateral carpal tunnel syndrome caused by an occult palmar lipoma. Orthopedics. 2009;32:777-779.

10. Morley GH. Intraneural lipoma of the median nerve in the carpal tunnel. Report of a case. J Bone Joint Surg Br. 1964;46:734-735. 